\title{
Matrix metalloproteinases and their natural inhibitors in fibrovascular membranes of proliferative diabetic retinopathy
}

Joel Salzmann, G Astrid Limb, Peng T Khaw, Zdenek J Gregor, Lynne Webster, Anthony H Chignell, David G Charteris

\begin{abstract}
Aim-To examine epiretinal membranes of proliferative diabetic retinopathy (PDR) for the presence of selective matrix metalloproteinases (MMPs) and their natural inhibitors (TIMPs), in order to determine whether neovascularisation and fibrosis, characteristic of this complication of diabetes mellitus, are associated with specific anomalies of MMP or TIMP expression.
\end{abstract}

Methods-The presence of selected MMPs and TIMPs was investigated in 24 fibrovascular epiretinal membranes of PDR, and the findings compared with that observed in 21 avascular epiretinal membranes of proliferative vitreoretinopathy (PVR) and five normal retinas. Specimens were examined for deposition of interstitial collagenase (MMP-1), stromelysin-1 (MMP-3), gelatinase A (MMP-2), gelatinase $B$ (MMP-9), and three tissue inhibitors of metalloproteinases (TIMP-1, TIMP-2, and TIMP-3).

Results-The results showed that unlike normal retina, which constitutively expresses MMP-1 and TIMP-2, a large proportion of PDR membranes ( $>62 \%$ ) stained for MMP-1, MMP-2, MMP-3, MMP-9, TIMP-1, TIMP-2, and TIMP-3. There were no differences in the expression of these molecules when compared with PVR membranes. A characteristic staining for MMP-9 was observed within the perivascular matrix of PDR membranes, and there was a significant increase in TIMP-2 expression by PDR membranes $(p=0.036)$ when compared with PVR membranes.

Conclusions-The findings that MMPs involved in degradation of fibrovascular tissue matrix, as well as TIMP-1 and TIMP-2, are found in a large proportion of PDR membranes, and that their expression does not differ from that of PVR membranes, suggest the existence of common pathways of extracellular matrix degradation in pathological processes leading to retinal neovascularisation and fibrosis.

(Br f Ophthalmol 2000;84:1091-1096)
Proliferative diabetic retinopathy (PDR) is a common complication of diabetes mellitus characterised by preretinal neovascularisation and development of epiretinal fibrovascular tissue. ${ }^{1}$ Early features of diabetic retinopathy include selective loss of intramural pericytes from retinal capillaries which leads to damage of the inner blood-retinal barrier. ${ }^{1}$ This process is followed by neovascularisation, involving the production of angiogenic factors, ${ }^{23}$ as well as synthesis of extracellular matrix (ECM) necessary for anchorage of migrating endothelium and other cells such as retinal pigment epithelium (RPE), glial cells, and fibroblasts. ${ }^{4}$ Degradation of ECM proteins is exerted by matrix metalloproteinases (MMPs), ${ }^{5}$ a family of zinc binding, calcium dependent enzymes, whose activity is in turn regulated by natural inhibitors known as tissue inhibitors of metalloproteinases (TIMPs). ${ }^{6}$ MMPs have an important role in connective tissue remodelling and in the degradation of basement membrane and surrounding extracellular matrix during processes of angiogenesis, ${ }^{78}$ while TIMPs are multifunctional proteins with both cell growth promoting and inhibitory activities. ${ }^{9}$ A balance between MMP and TIMP activities may preserve tissue integrity, while excessive MMP or TIMP production may lead to excessive degradation or increased accumulation of extracellular matrix. ${ }^{56}$ It is therefore possible that an imbalance between MMPs and TIMPs may lead to the neovascularisation that characterises the development of retinal abnormalities in insulin dependent diabetes mellitus (IDDM), and to the fibrocellular proliferation observed in neovascular and avascular retinopathies.

Increased levels of MMP-9, a type IV collagenase implicated in tumour angiogenesis, ${ }^{10}$ are found in vitreous from eyes with $\mathrm{PDR}^{11}$ and PVR, ${ }^{12}{ }^{13}$ and retinal membranes of proliferative vitreoretinopathy (PVR) stain for MMPs $1,2,3$, and 9, as well as TIMP-1 and TIMP-2. ${ }^{14}$ This contrasts with the expression of MMP-1 and TIMP-2 but not other MMPs by normal retina. ${ }^{14}$ Although PVR and PDR have different aetiologies and clinical characteristics, retinal membranes from both conditions share the features of fibroplasia, excessive 
matrix protein deposition, and cellular infiltration. ${ }^{15}$ They differ in that PDR membranes are highly vascular, owing to the angiogenic activity that takes place within the diabetic retina, ${ }^{16}$ while PVR membranes are relatively avascular ${ }^{17}$ and are not regarded as a complication of systemic disease. At present it is not known whether retinal tissues from eyes with PDR express MMPs or TIMPs associated with neovascularisation, or if there are differences in the expression of these molecules between epiretinal membranes of PDR and PVR.

The aim of this study was to examine PDR membranes for the presence of MMPs and TIMPs potentially responsible for neovascularisation and fibrosis, and to compare their expression with that observed in avascular retinal membranes from nondiabetic patients and normal retina. On this basis we compared PDR and PVR membranes for the presence of interstitial collagenase (MMP-1), stromelysin-1 (MMP-3), gelatinase A (MMP-2), gelatinase B (MMP-9), and their natural inhibitors TIMP-1, TIMP-2, and TIMP-3.

\section{Experimental tissue and methods}

Epiretinal membranes were obtained from 24 eyes undergoing pars plana vitrectomy for treatment of diabetic tractional retinal detachment, and from 21 eyes with rhegmatogenous retinal detachment complicated by PVR. Normal retina was obtained from five cornea donor eyes 12-24 hours post mortem. Four membranes derived from patients with noninsulin dependent diabetes mellitus (NIDDM). The study was performed in accordance with the ethical standards laid down in the 1964 Declaration of Helsinki. Membranes were collected in 4\% paraformaldehyde in phosphate buffered saline (PBS) and fixed for 1-2 hours. After immersion for 1 hour in $15 \%$ sucrose in PBS, tissue was embedded in OCT compound (BDH, UK) and stored in liquid nitrogen until use. Serial cryostat sections ( $7 \mu \mathrm{m}$ thick) were placed on slides coated with poly-L-lysine $(0.01 \% \mathrm{w} / \mathrm{v}$ : Sigma, UK), fixed in $4 \%$ paraformaldehyde for 5 minutes followed by $15 \%$ sucrose for 10 minutes, and processed for immunohistochemistry.

\section{IMMUNOHISTOCHEMICAL DETECTION OF MMPS} AND TIMPS

The presence of MMPs and TIMPs was determined by immunohistochemical staining using the alkaline phosphatase anti-alkaline phosphatase detection system, performed according to our published methods. ${ }^{14}{ }^{18}$ Tissue sections were washed in TRIS buffered saline $\mathrm{pH} 7.4$ (0.05M TRIS in $0.15 \mathrm{NaCl}$ TBS) and incubated for 3 hours with a panel of commercially available mouse monoclonal antibodies to selected human MMPs and TIMPs (Oncogene research products: CalbiochemNovabiotechem International, Nottingham, UK). All antibodies were diluted in $0.5 \%$ blocking reagent (Boehringer Mannheim) in TBS to a final concentration of $10 \mu \mathrm{g} / \mathrm{ml}$. These included anti-MMP-1 (clone 41-1E5, detecting active and latent forms), antiMMP-2 (clone 42-5D11, detecting latent form), anti-MMP-3 (clone 55-2A4, detecting active and latent form), anti-MMP-9 (clone $6-6 \mathrm{~B}$, detecting active and latent forms), anti-TIMP-1 (clone 7-6C1), anti TIMP-2 (clone T2-101), and anti-TIMP-3 (clone 13613H4). These antibodies are known to be specific for the above molecules as previously confirmed by zymography ${ }^{19}$ and to identify epitopes that are preserved by fixation with PFA. ${ }^{14}$ Mouse total IgG antibody (Sigma, UK) was used as a negative control in the assay. Non-specific binding of the antibodies to constitutive proteins of retinal membranes was excluded by the fact that not all membranes stained with each of the antibodies used in the study.

Table 1 Presence of selected matrix metalloproteinases (MMPs) and their natural inhibitors in PDR membranes

\begin{tabular}{|c|c|c|c|c|c|c|c|c|c|}
\hline Membrane No & $\begin{array}{l}\text { Type of } \\
\text { diabetes }\end{array}$ & $M M P-1$ & $M M P-2$ & $M M P-3$ & $M M P-9$ & $T I M P-1$ & $T I M P-2$ & TIMP-3 & Total mouse IgG \\
\hline 2 & IDDM & +++ & - & - & - & - & + & ND & - \\
\hline 3 & IDDM & - & - & - & + & + & - & ND & - \\
\hline 4 & IDDM & +++ & - & - & ++ & + & + & ++ & - \\
\hline 5 & IDDM & - & - & + & - & - & - & ND & - \\
\hline 6 & IDDM & + & ++ & ++ & ++ & + & - & + & - \\
\hline 7 & IDDM & - & - & - & - & - & - & ND & - \\
\hline 8 & IDDM & +++ & - & ++ & +++ & + & + & - & - \\
\hline 9 & IDDM & - & - & - & - & - & - & ND & - \\
\hline 10 & IDDM & - & + & ++ & ++ & + & + & ND & - \\
\hline 11 & IDDM & + & + & ++ & ++ & ++ & + & + & - \\
\hline $12^{\mathrm{a}}$ & IDDM & +++ & +++ & +++ & +++ & ++ & ++ & ND & - \\
\hline $13^{\mathrm{b}}$ & IDDM & - & ++ & - & +++ & - & - & - & - \\
\hline 15 & IDDM & +++ & +++ & + & + & ++ & + & ND & - \\
\hline 16 & IDDM & ++ & ++ & - & - & - & + & ND & - \\
\hline 17 & IDDM & ++ & + & +++ & + & ++ & + & ++ & - \\
\hline 19 & IDDM & +++ & +++ & +++ & +++ & ++ & ++ & ++ & - \\
\hline 21 & IDDM & +++ & + & + & - & - & + & - & - \\
\hline 22 & IDDM & ++ & ++ & - & +++ & ++ & + & - & - \\
\hline $23^{\mathrm{c}}$ & IDDM & - & + & - & + & + & - & ND & - \\
\hline $24^{\mathrm{d}}$ & IDDM & - & - & + & - & + & - & ND & - \\
\hline 1 & NIDDM & +++ & ++ & ++ & ++ & + & - & ++ & - \\
\hline 14 & NIDDM & +++ & +++ & +++ & - & +++ & + & ND & - \\
\hline 18 & NIDDM & +++ & +++ & ++ & +++ & ++ & + & ++ & - \\
\hline 20 & NIDDM & ++ & ++ & +++ & +++ & ++ & + & + & - \\
\hline
\end{tabular}

[a-b]Same patient; left and right eye respectively; [c-d] same patient, right and left eye respectively. Intensity of staining: $+++=$ strong,$++=$ moderate, $+=$ mild, $-=$ negative, $\mathrm{ND}=$ not done. 

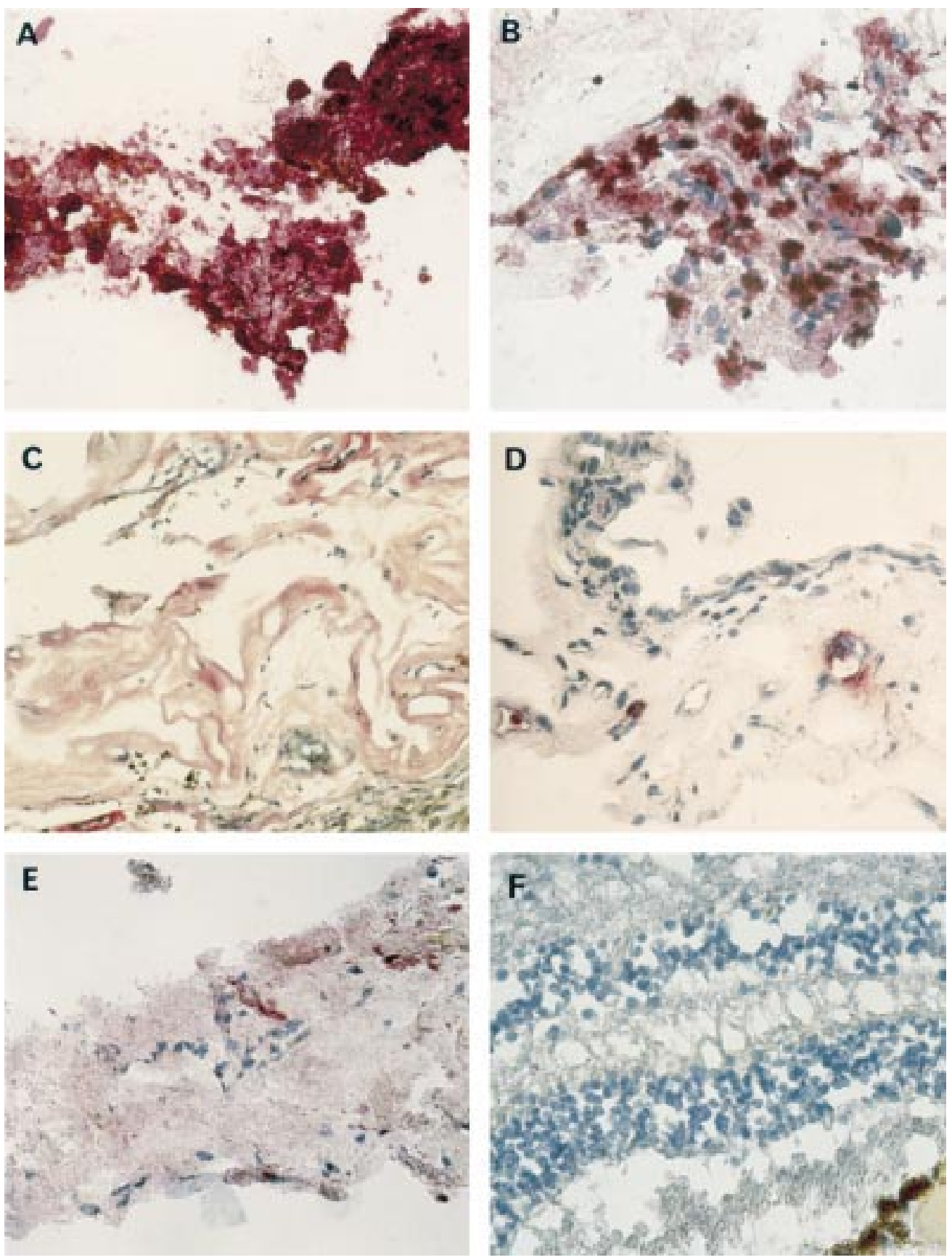

Figure 1 Photomicrographs of PDR membranes showing deposition of selective MMPs and TIMPs. The pink precipitate indicates the presence of these molecules within the membranes. Specimens were counterstained with Mayer's haematoxylin. (A) PDR membrane (No 15) showing strong staining (+++) for MMP-1. (B) PDR membrane (No 12) showing intense cellular and extracellular staining (+++) for MMP-2. (C) PDR membrane (No 1) exhibiting staining for MMP-9 within the extracellular and perivascular matrix. (D) Section of PDR membrane (No 17) showing detail staining for MMP-9 in the perivascular matrix. (E) PDR membrane exhibiting moderate staining (++) for TIMP-1 (No 11). (F) Normal retina showing negative staining for MMP-9.

After incubation with the primary antibody, specimens were washed for 2 minutes in TBS, incubated for 30 minutes with $50 \mu \mathrm{l}$ rabbit anti-mouse antibody (Dako, UK), and further washed for 2 minutes in TBS. Sections were then incubated with $50 \mu 1$ monoclonal mouse alkaline phosphatase anti-alkaline phosphatase antibodies (Dako) for 30 minutes and washed for 2 minutes. The last two steps were repeated for 10 minutes each to enhance staining intensity, followed by incubation for 30 minutes with developing substrate $(1 \mathrm{mg} / \mathrm{ml}$ fast red in $0.1 \mathrm{M}$ TRIS buffer pH 8.2 with $1.2 \mathrm{mM}$ naph- thol AS-MX phosphate, 0.25 dimethylformamide, and $1 \mathrm{mM}$ levamisole). Specimens were finally washed in distilled water, counterstained with Mayer's haematoxylin, and mounted with glycergel mounting medium (Dako).

Membranes were examined under light microscopy for positive staining identified by a red precipitate. Intensity of staining was graded by two independent observers into four categories: negative $(-)$, mild $(+)$, moderate $(++)$, and strong $(+++)$, according to the strength and distribution of the red precipitate. 
Table 2 Comparison between retinal membranes of PDR and PVR and normal retina in the expression of MMPs and TIMPs

\begin{tabular}{lccllrrr}
\hline & $\begin{array}{l}M M P-1 \\
\text { (active + latent) }\end{array}$ & $\begin{array}{l}\text { MMP-2 } \\
\text { (latent) }\end{array}$ & $\begin{array}{l}\text { MMP-3 } \\
\text { (active + latent) }\end{array}$ & $\begin{array}{l}\text { MMP-9 } \\
\text { (active +latent) }\end{array}$ & TIMP-1 & TIMP-2* & TIMP-3 \\
\hline PDR & $66 \%$ & $66 \%$ & $62 \%$ & $66 \%$ & $71 \%$ & $62 \%$ \\
PVR & $86 \%$ & $81 \%$ & $52 \%$ & $48 \%$ & $43 \%$ & $33 \%$ & $75 \%$ \\
Normal retina & $100 \%{ }^{\mathrm{a}}$ & $0 \%$ & $0 \%$ & $0 \%$ & $0 \%$ & $100 \%$ & $0 \%$ \\
\hline
\end{tabular}

The figures represent the percentage of samples staining for each antibody in the different groups.

${ }^{\star} \mathrm{p}=0.036 v$ PVR

a Staining of the inner and outer nuclear cell layers and plexiform layers.

${ }^{\mathrm{b}}$ Staining of the inner and outer nuclear cell layers only.

STATISTICAL ANALYSIS

The significance of difference between corresponding groups of observations was evaluated by the non-parametric Fisher's exact test. Acceptable significance was recorded when $\mathrm{p}$ values were $<0.05$

\section{Results}

EXPRESSION OF MMPS AND TIMPS IN PDR MEMBRANES

Table 1 shows the distribution of MMPs and TIMPs within the extracellular matrix of epiretinal membranes of PDR. The majority of membranes $(22 / 24,92 \%)$ stained for at least one of the MMPs or TIMPs examined, while two of the membranes did not show deposition of any of the molecules investigated. None of the specimens stained with all antibodies to MMPs and TIMPs used in this study. Of the 24 membranes analysed, $16(66 \%)$ stained for interstitial collagenase (MMP-1) (Fig 1A), 16 $(66 \%)$ for gelatinase A (MMP-2) (Fig 1B), and $15(62 \%)$ for stromelysin-1 (MMP-3). Expression of gelatinase B (MMP-9) was observed in 16 membranes (66\%), some of which showed staining of the perivascular extracellular matrix (Figs 1C and D). Presence of TIMP-1 (Fig 1D) and TIMP-2 was observed in $17(71 \%)$ and $15(62 \%)$ membranes respectively, and the intensity of staining for TIMP-1 was in general greater than that for TIMP-2 (Table 1). TIMP-3 was observed in 8/12 (66\%) membranes and none of the membranes investigated stained with total mouse IgG (Table 1).

Of the 16 specimens stained for MMP-1, 13 were also positive for MMP-2, 12 for MMP-3, 12 for MMP-9, 13 for TIMP-1, and 14 for TIMP-2 (Table 1). All membranes staining for TIMP-3 (eight) were also positive for TIMP-1 and MMP-1, and six were positive for TIMP-2. Of the 15 membranes staining for MMP-3, 10 also stained for both MMP-2 and MMP-9, and 12 stained for TIMP-1 (Table 1). Thirteen of the 16 membranes positive for MMP-9 also stained for MMP-2, 15 for TIMP-1, and 11 for TIMP-2. Twelve of the 17 specimens positive for TIMP-1 also stained for TIMP-2 (Table 1), but overall, the intensity of staining for TIMP-1 was higher than that for TIMP-2 (Table 1). The strongest staining for any of the molecules investigated was observed with antibodies to MMP-1, and no differences in the staining for MMPs and TIMPs were evident when comparing membranes from patients with IDDM and NIDDM. Four of PDR membranes that derived from two patients who underwent pars plana vitrectomy in their left and right eyes on different occasions did not show the same pattern of staining for the MMPs and TIMPs investigated (Table 1).

COMPARISON BETWEEN EPIRETINAL MEMBRANES OF PDR AND PVR AND NORMAL RETINA IN THE EXPRESSION OF MMPS AND TIMPS

There was no significant difference between the proportion of PDR membranes staining for MMP-3 (15/24) and MMP-9 (16/24) when compared with PVR membranes (11/21 and $10 / 21$, respectively; $p>0.5$ ) (Table 2 ). The proportion of PDR membranes staining for TIMP-1 (17/24) was slightly higher than that of PVR membranes staining for this molecule $(9 / 21)$, but this difference was not significant $(p$ $=0.075)$. However, the percentage of PDR membranes staining for TIMP-2 (15/24) was significantly higher when compared with that of PVR membranes $(7 / 21 ; p=0.036)$. A similar proportion of TIMP-3 positive membranes was observed in both PDR (8/12) and PVR (15/20).

As previously shown, ${ }^{14}$ normal retina did not express MMP-9 (Fig 1F, Table 2), but stained for MMP-1 in the inner and outer nuclear and plexiform layers, as well as for TIMP-2 in both the inner and outer nuclear layers (Table 2).

\section{Discussion}

This study shows that a large proportion of PDR membranes (92\%) exhibited deposition of MMPs potentially responsible for degrading matrix constituents of blood vessels and epiretinal membranes. In addition, a large percentage of PDR membranes (75\%) also expressed TIMPs, which by inhibiting matrix degradation by MMPs may promote its accumulation with resultant fibrosis and new vessel formation. The proportion of membranes staining for the MMPs investigated was similar in both PDR and PVR, suggesting that the same molecular mechanisms of extracellular matrix degradation and deposition may operate in vascular and avascular proliferative processes affecting the retina.

Since MMP-1 is constitutively expressed in normal retina, ${ }^{14}$ its presence in retinal membranes of both PVR and PDR could be anticipated. It is possible that this MMP may have a physiological role within the retina, such as aiding degradation of the shed tips of photoreceptors, and this merits further investigation. Membrane expression of MMP-2, MMP-3, and MMP-9, which are not observed in normal retina may indicate an active involvement of these molecules in the neovascularisation observed in PDR and in the fibrocellular proliferation observed in both PDR and PVR. Early vascular changes in diabetic retinopathy 
include the loss of pericytes ${ }^{1}$ and it has been postulated that this phenomenon may be facilitated by MMP degradation of basement membrane and surrounding matrix. ${ }^{78}$ In this context, our observation that matrix surrounding the vessels of PDR membranes stains for MMP-9 (Fig 1C, D), strongly suggests that this molecule, which degrades denatured collagens and type IV basement membrane collagen, may contribute to retinal neovascularisation in PDR. That MMP-9 may be actively involved in diabetic retinal neovascularisation is supported by observations that high vitreous levels of this molecule may be found in eyes with PDR, ${ }^{11}{ }^{12}$ and that microvascular endothelial permeability is increased in the presence of this $\mathrm{MMP} .^{20}$ This is further supported by the demonstration that levels of expression of MMP-9 correlate with tumour neovascularisation in vivo, ${ }^{10}$ and that increased plasma levels of this molecule precede the development of renal microvascular complications in patients with IDDM. ${ }^{21}$ This is of special interest, as renal microangiopathy is often associated with proliferative retinopathy in diabetic individuals. ${ }^{22}$

This study showed a slight but not statistically significant increase in the percentage of PDR membranes staining for TIMP-1 (70\%) when compared with PVR membranes (42\%; p $=0.06)$. It contrast, there was a significant increase in the proportion of PDR membranes staining for TIMP-2 (62\%) when compared with PVR membranes $(33 \%$; $p=0.036)$. These observations suggest that differences in the kinetic mechanisms of TIMP production and activity within the retina may determine the development of different patterns of matrix accumulation, and therefore whether new vessels are formed during a pathological process. Although TIMP-1 and TIMP-2 inhibit angiogenesis and exhibit growth factor-like activity, ${ }^{9}$ it is difficult to speculate on their specific role in the process of neovascularisation in the diabetic retina. It may be possible that these molecules are produced to control MMP activity, but if excessive production of MMPs occurs, or insufficient levels of TIMPs are released, they may not exert an adequate anti-angiogenic activity.

It is unlikely that the MMPs and TIMPs observed in PDR membranes derived from the microvascular compartment, as there were no differences with that observed in membranes from eyes with PVR, in which no vitreous haemorrhage was present and in which neovascularisation does not occur. However, it is possible that these molecules may be locally produced by cells of the retinal microenvironment, such as RPE and glial cells, which are known to produce various MMPs and TIMPs. ${ }^{23-25}$

The mechanisms that trigger release of MMPs and TIMPs during retinal neovascularisation are not known, but it is conceivable that cytokines produced upon endothelial cell activation by ischaemia may induce local release of these molecules. It is suggested by observations that cytokines such as tumour necrosis factor $\alpha(\mathrm{TNF} \alpha)$ and interleukin-1
(IL-1), which are found in vitreous ${ }^{26}$ and membranes ${ }^{18}$ from eyes with PDR, induce production of MMPs and TIMPs by vascular endothelial cells, fibroblasts, and retinal pigment epithelial cells. ${ }^{24252728}$ This is of special relevance to the pathogenesis of PDR, as there is an association between susceptibility to retinopathy and $\mathrm{TNF} \alpha$ polymorphism in individuals with NIDDM, ${ }^{29}$ and subjects with IDDM expressing the TNF2 allele produce high levels of $\mathrm{TNF} \alpha{ }^{30}$

The observations that MMPs and TIMPs implicated in neovascularisation and fibrosis are found within PDR and PVR membranes, indicate that although both conditions have different aetiologies and clinical characteristics, these molecules may play an important part in the pathogenesis of both conditions. Further studies may elucidate whether deposition of MMP-9 in the perivascular matrix is unique to PDR, or whether it is a common feature of all microvascular complications of diabetes mellitus. From the present findings it is not clear whether there are selective defects in the balance between MMPs and TIMPs which either promote fibrosis and neovascularisation in PDR, or fibrosis in PVR. However, investigation of the kinetics of production of selective MMPs and their control by specific TIMPs may aid in the design of new therapeutic approaches to treat and prevent PDR and other microvascular complications of diabetes mellitus.

Supported by the Henry Smith Charity.

1 Garner A. Histopathology of diabetic retinopathy in man. Eye 1993;7:250-3.

2 Forrester JV, Shafiee A, Schröder S, et al. The role of growth factors in proliferative diabetic retinopathy. Eye 1993;7: 276-87.

3 Adamis AP, Miller JW, Bernal M-T, et al. Increased vascular endothelial growth factor levels in the vitreous of eyes with proliferative diabetic retinopathy. Am f Ophthalmol 1994; 118:445-50.

4 Hiscott P, Waller HA, Grierson I, et al. The extracellular matrix of reparative tissue in the vitreous: fibronectin production in proliferative diabetic retinopathy membranes. Eye 1993;7:288-92.

5 Matrisian LM. The matrix-degrading metalloproteinases. Bio Assays 1992;14:455-63.

6 Dollery CM, McEwan JR, Henney AM. Matrix metalloDollery CM, McEwan JR, Henney AM. Matrix metallo-
proteinases and cardiovascular disease. Circ Res 1995;77: 863-8.

7 Taylor CM, Thompson JM, Weiss JB. Matrix integrity and the control of angiogenesis. Int f Radiat Biol 1991;60:61-4.

8 Furness PN. Basement membrane synthesis and degradation. F Pathol 1997;183:1-3.

9 Gomez DE, Alonso DF, Yoshiji H, et al. Tissue inhibitors of metalloproteinases: structure, regulation and biological functions. Eur F Cell Biol 1997;74:111-22.

10 Kurizaki T, Toi M, Tominaga T. Relationship between matrix metalloproteinase expression and tumor angio-
genesis in human breast carcinoma. Oncol Rep 1998;60: genesis

11 Brown D, Hamdi H, Bahri S, et al. Characterization of an endogenous metalloproteinase in human vitreous. Curr Eye Res 1994;13:639-47.

12 De La Paz MA, Itoh Y, Toth CA, et al. Matrix metalloproteinases and their inhibitors in human vitreous. Invest Ophthalmol Vis Sci 1998;39:1256-60.

13 Kon CH, Occleston NL, Charteris DG, et al. A prospective study of matrix metalloproteinases in proliferative vitreoretinopathy. Invest Ophthal Vis Sci 1998;39:1524-9.

14 Webster L, Chignell AH, Limb GA. Predominance of MMP-1 and MMP-2 in epiretinal and subretinal membranes of proliferative vitreoretinopathy. Exp Eye Res 1999; 68:91-8.

15 Gilbert C, Hiscott P, Unger W, et al. Inflammation and the formation of epiretinal membranes. Eye 1988;(Suppl)2: S140-56.

16 Hiscott P, Waller HA, Grierson I, et al. The extracellular matrix of reparative tissue in the vitreous: fibronectin production in proliferative diabetic retinopathy membranes. Eye 1993;7:288-92. 
17 Jerdan JA, Pepose JS, Michels RG, et al. Proliferative vitreoretinopathy membranes. An immunocytochemical study. Ophthalmology 1989;96:801-10.

$18 \mathrm{Limb} \mathrm{GA}$, Chignell AH, Green W, et al. Distribution of $\mathrm{TNF} \alpha$ and its reactive vascular adhesion molecules in fibrovascular membranes of proliferative diabetic retinopathy. Br f Ophthalmol 1996;80:168-73.

19 Guo L, Hussain AA, Limb GA, et al. Age-dependent variation in metalloproteinase activity of isolated human Bruch's membrane and choroid. Invest Ophthalmol Vis Sci 1999;40:2676-82.

20 Partridge CA, Jeffrey JJ, Malik AB. A 96kDa gelatinase induced by TNF-alpha contributes to increased microvascular endothelial permeability. Am f Physiol 1993;265: L438-47.

21 Ebihara I, Nakamura T, Shimada N, et al. Increased plasma metalloproteinase-9 concentrations precede development of microalbuminuria in non-insulin dependent diabetes mellitus. Am 7 Kidney Dis 1998;32:544-50.

22 Skyler JS. Diabetic complications. Endocrin Metab N Am 1996;25:243-55.

23 Hunt RC, Fox A, Pakalnis VA, et al. Cytokines cause cultured retinal pigment epithelial cells to secrete metalloproteinases and to contract collagen gels. Invest Ophthalmol Vis Sci 1993;34:3179-86.
24 Alexander JP, Bradley JMB, Gabourel JD, et al. Expression of matrix metalloproteinases and inhibitor by human retinal pigment epithelium. Invest Ophthalmol Vis Sci 1990;

25 Gottschall PE, Yu X. Cytokines regulate gelatinase A and B (matrix metalloproteinase 2 and 9) activity in cultured rat astrocytes. F Neurochem 1995;64:1513-20.

26 Franks WA, Limb GA, Stanford MR, et al. Cytokines in human intra-ocular inflammation. Curr Eye Res 1992;11: 187-91.

27 Grant MB, Caballero S, Tarnuzzer RW, et al. Matrix metalloproteinase expression in human retinal microvascular cells. Diabetes 1998;47:1311-17.

28 Schwartz DJ, Monea S, Marcus SG, P et al. Soluble factor(s) released from neutrophils activates endothelial cell matrix metalloproteinase-2. F Surg Res 1998;76:79-85.

29 Hawrami K, Hitman GA, Rema M, et al. An asssociation in non-insulin-dependent diabetes mellitus subjects between susceptibility to retinopathy and tumor necrosis factor polymorphism. Human Immunol 1996;46:49-54.

30 Pociot F, Briant L, Jongeneel CV, et al. Association of tumor necrosis factor (TNF) and class II major histocompatibility necrosis factor (TNF) and class II major histocompatibility alleles with secretion of TNF $\propto$ and TNF $\beta$ by human
monoculear cells: a possible link to insulin-dependent diabetes mellitus. Eur f Immunol 1993;23:224-31.

\section{Contributors please note:}

Communications from all countries except the UK and Republic of Ireland should be sent to Professor C Hoyt, Editor, British fournal of Ophthalmology, University of California, Department of Ophthalmology, 10 Kirkham Street, K 301, San Francisco, CA 94143-0730, USA (tel: 001415 502-6871; fax: 001415 514-1521).

Manuscripts from the UK and the Republic of Ireland should be sent to Professor Andrew Dick, UK Editor, British fournal of Ophthalmology, Division of Ophthalmology, Unversity of Bristol, Lower Maudlin Street, Bristol BS1 2LX (tel: +44 (0) 0117 929-4496; fax: +44 (0) 117 929-4607). 